International Journal of Applied Mathematics

Volume 30 No. $3 \quad 2017,211-228$

ISSN: $1311-1728$ (printed version); ISSN: 1314-8060 (on-line version)

doi: http://dx.doi.org/10.12732/ijam.v30i3.1

\title{
NUMERICAL APPROXIMATION OF SPECTRUM FOR VARIABLE COEFFICIENTS EULER-BERNOULLI BEAMS UNDER A FORCE CONTROL IN POSITION AND VELOCITY
}

\author{
Kouassi Ayo Ayébié Hermith ${ }^{1}$, Coulibaly Adama², \\ Touré Kidjégbo Augustin ${ }^{3}$ \\ ${ }^{1}$ Université Félix Houphouët Boigny \\ Yamoussoukro, BP 2444, CÔTE D'IVOIRE \\ ${ }^{2}$ Université Félix Houphouët Boigny \\ BP 582, Abidjan 22, CÔTE D'IVOIRE \\ ${ }^{3}$ Institut National Polytechnique Houphouët-Boigny \\ Yamoussoukro, BP 2444, CÔTE D'IVOIRE
}

\begin{abstract}
In this paper, we use asymptotic techniques and the finite differences method to study the spectrum of differential operator arising in exponential stabilization of Euler-Bernoulli beam with nonuniform thickness or density that is clamped at one end and is free at the other. To stabilize the system, we apply at the free end, the following shear force feedback control:
\end{abstract}

$$
\left(E I(\cdot) u_{x x}(\cdot, t)\right)_{x}(1)=\alpha u(1, t)+\beta u_{t}(1, t), \quad t>0 .
$$

We build a numerical scheme and investigate the eigenvalues locus as a function of the positive feedback parameters $\alpha$ and $\beta$.

AMS Subject Classification: 93C20, 93D15, 35B35, 35P10

Key Words: numerical approximation problem, semigroup theory, asymptotic behavior, exponential stability, finite differences

Received: March 9, 2017

(c) 2017 Academic Publications

${ }^{\S}$ Correspondence author 


\section{Introduction}

Consider the following evolutive system:

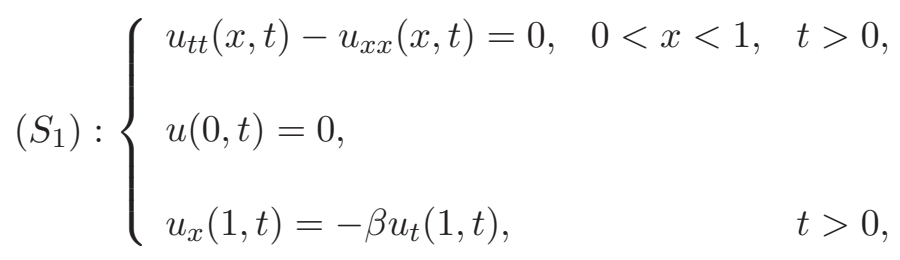

where $u$ is a scalar function of variables $x$ and $t, \beta$ is a nonzero positive constant.

This simplified model represents for example a cable clamped at one end and is submitted to a linear boundary control force in velocity at the free end. The cable is supposed flexible with constant length.

Many authors have studied the above system (see [5] for example and the references therein) and have proved that the system $\left(S_{1}\right)$ is exponentially stable for all $\beta \neq 1$. Moreover, they have proved that the system $\left(S_{1}\right)$ verifies the Riesz basis property and obtained the spectrum by an explicit formula.

The idea of adding a control force in position to the existing feedback has been invoked by the studies of many authors (see [4]).

Consider the following system:

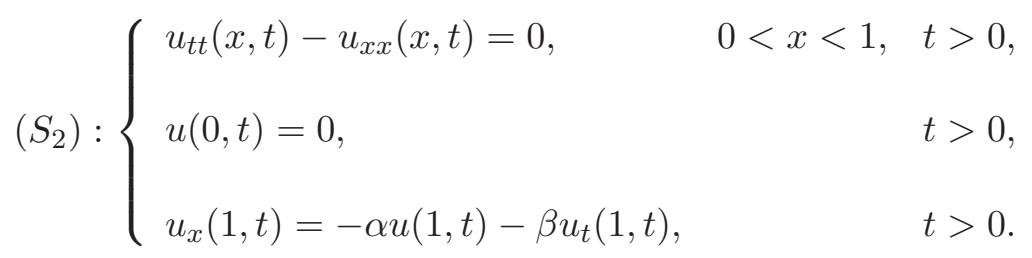

From mathematical point of view one wants to check if the properties of the disrupted system stay intact. To this question many authors have provided a positive answer. The system $\left(S_{1}\right)$ obtained by disruption of system $\left(S_{1}\right)$ is exponentially stable again (see [10]) and verifies the Riesz basis property (see $[14])$.

From practical point of view, the goal is to improve the optimal decay rate of energy. To this practical preoccupation which takes its importance from cost of the realization of models thus obtained theoretically, it should provide a satisfactory answer. The theoretical study of such problem, is not easy even for a simple model like the system $\left(S_{1}\right)$.

In ([4] and [10]) the authors proved that the fact of adding a control force in position to existing control in velocity, although preserving the Riesz basis 
property and the exponential stability has negative effect on the optimal decay rate of elastic energy.

In [8] the authors use the finite differences method and the QZ method to describe geometrically the spectrum and have got the same results.

So the idea of using numerical methods to study the impact of adding a control to an existing control, seems to be a credible alternative.

In this paper we consider the evolutive system given by:

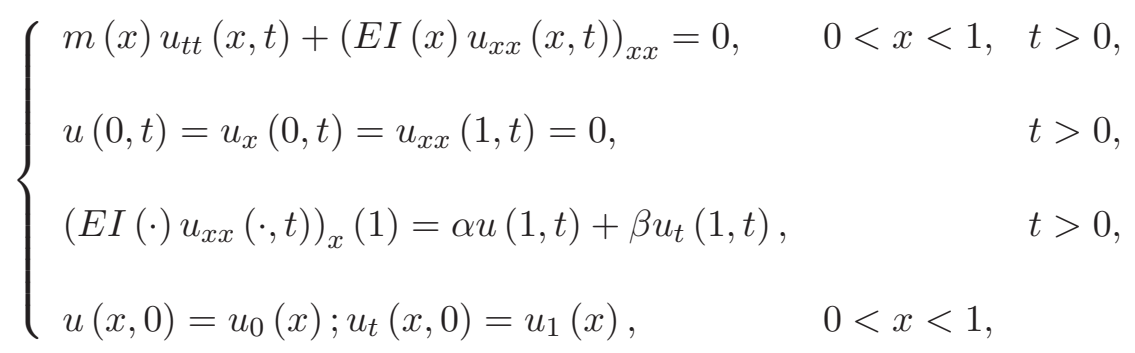

where $\alpha, \beta$ are two given positive constants, $u(x, t)$ stands for a transversal deviation of the beam at position $x$ and time $t$, a subscript letter denotes the partial derivation with respect that variable. The length of the beam is chosen to be unity, $E I($.$) is the stiffness of the beam, and m($.$) is the mass density.$ Moreover, we shall always assume that:

$$
m(\cdot), E I(.) \in C^{4}(0,1) \quad \text { and } \quad m(x), E I(x)>0 .
$$

For $\alpha=0$, many authors have proved the Riesz basis property and the exponential stability (see [2], [12]).

In this paper one uses the finite differences method and the QZ method to answer the following question: does the control in position improve the optimal decay rate of elastic energy? The main goal of this work is to use the finite differences method to elaborate a program that gives the complete eigenvalues location of the system defined by (3), as a function of positive feedback parameters $\alpha$ and $\beta$.

The paper is organized as follows. In Section 2 we recall the formulation of the system (3) in the context of $\mathrm{C}_{0}$-semigroup of contractions theory. Then, in Section 3 we formulate the eigenvalue problem so that to use QZ method. In Section 4, we develop a numerical scheme based on the finite differences method for the eigenvalues problem. Finally, in the last section, we give some numerical experiments from our scheme to answer at the question. 


\section{Formulation of the System (3) in the Context of the $\mathrm{C}_{0}$-Semigroup of Contractions Theory}

Let us introduce the following spaces:

$$
\begin{gathered}
V_{E}^{2}=\left\{u(x) \in H^{2}(0,1): u(0)=u_{x}(0)=0\right\}, \\
\mathbb{H}=V_{E}^{2}(0,1) \times L^{2}(0,1),
\end{gathered}
$$

$D(0,1)=$ the space of smooth functions with compact support, $D^{\prime}(0,1)=$ the space of continuous linear functions, $f: D(0,1) \rightarrow \mathbb{C}$.

The superscript $T$ stands for the transpose and the spaces $L^{2}(0,1)$ and $H^{k}(0,1)$ are defined as

$$
\begin{gathered}
L^{2}(0,1)=\left\{u:[0,1] \rightarrow \mathbb{R}: \int_{0}^{1} u^{2} d x<\infty\right\} \\
H^{k}(0,1)=\left\{u:[0,1] \rightarrow \mathbb{R}: u, u^{(1)}, \ldots, u^{(k)} \in L^{2}(0,1)\right\} .
\end{gathered}
$$

In the space $\mathbb{H}$, we define the inner-product

$$
\begin{aligned}
\langle u, v\rangle_{\mathbb{H}}= & \int_{0}^{1}\left(m(x) f_{2}(x) \overline{g_{2}(x)}+E I(x) f_{1}^{\prime \prime}(x) \overline{g_{1}^{\prime \prime}(x)}\right) d x \\
& +\alpha f_{1}(1) \overline{g_{1}(1)}
\end{aligned}
$$

where $u=\left(f_{1}, f_{2}\right)^{T} \in \mathbb{H}$ et $u=\left(g_{1}, g_{2}\right)^{T} \in \mathbb{H}$.

Next, we define an unbounded linear operator $\mathbb{A}: D(\mathbb{A}) \subset \mathbb{H} \rightarrow \mathbb{H}$ as follows:

$$
\mathbb{A}(f, g)=\left(g(x),-\frac{1}{m(x)}\left(E I(x) f^{\prime \prime}(x)\right)^{\prime \prime}\right),
$$

where $D(\mathbb{A})$, the domain of operator, is

$$
\begin{aligned}
& D(\mathbb{A})=\left\{(f, g)^{T} \in H^{4}(0,1) \cap V_{E}^{2}(0,1) \times V_{E}^{2}(0,1):\right. \\
& \left.f^{\prime \prime}(1)=0, \quad\left(E I(\cdot) f^{\prime \prime}(\cdot)\right)^{\prime \prime}(1)=\alpha f(1)+\beta g(1)\right\} .
\end{aligned}
$$

With these notations, the set of system (3) can be formally written as

$$
\left\{\begin{array}{l}
\frac{d Y(t)}{d t}=\mathbb{A} Y(t), \\
Y(0)=Y_{0} \in \mathbb{H},
\end{array}\right.
$$

where $Y(t)=\left(u(\cdot, t), u_{t}(\cdot, t)\right) ; Y(0)=\left(u_{0}, u_{1}\right)$. 
Theorem 1. The operator $\mathbb{A}$, defined by (10) and (11), generates a $C_{0}$-semigroup of contractions on $\mathbb{H}$.

Proof. See [13].

\section{Spectrum of Operator $\mathbb{A}$}

Now we are ready to study the eigenvalue problem of $\mathbb{A}$.

Let $\lambda \in \sigma(\mathbb{A})$ and $\Phi=(\phi, \Psi)$ be an eigenfunction of $\mathbb{A}$ corresponding to $\lambda$. Then we have $\Psi=\lambda \phi$ and $\phi$ satisfies the following equation:

$$
\left\{\begin{array}{l}
\lambda^{2} m(x) \phi(x)+\left(E I(x) \phi^{\prime \prime}(x)\right)^{\prime \prime}=0, \quad 0<x<1, \\
\phi(0)=\phi^{\prime}(0)=\phi^{\prime \prime}(1)=0, \\
\phi^{\prime \prime \prime}(1)=\frac{1}{E I(1)}(\alpha+\beta \lambda) \phi(1) .
\end{array}\right.
$$

Expanding (13) yields for all $0<x<1$,

$$
\left\{\begin{array}{l}
\phi^{(4)}(x)+\frac{2 E I^{\prime}(x)}{E I(x)} \phi^{\prime \prime \prime}(x)+\frac{E I^{\prime \prime}(x)}{E I(x)} \phi^{\prime \prime}(x)+\frac{\lambda^{2} m(x)}{E I(x)} \phi(x)=0, \\
\phi(0)=\phi^{\prime}(0)=\phi^{\prime \prime}(1)=0 \\
\phi^{\prime \prime \prime}(1)=\frac{1}{E I(1)}(\alpha+\beta \lambda) \phi(1) .
\end{array}\right.
$$

In order to simplify our computations, we introduce a spatial scale transformation in $x$ :

$$
f(z)=\phi(x), \quad z(x)=\frac{1}{p} \int_{0}^{x}\left(\frac{m(\zeta)}{E I(\zeta)}\right)^{\frac{1}{4}} d \zeta
$$

where

$$
p=\int_{0}^{1}\left(\frac{m(\zeta)}{E I(\zeta)}\right)^{\frac{1}{4}} d \zeta
$$


Then, (14) together with its boundary conditions can be transformed into

$$
\left\{\begin{array}{l}
f^{(4)}(z)+a(z) f^{\prime \prime \prime}(z)+b(z) f^{\prime \prime}(z)+c(z) f^{\prime}(z)+\lambda^{2} p^{4} f(z)=0 \\
0<z<1 \\
f(0)=f^{\prime}(0)=0 \\
z_{x}^{2}(1) f^{\prime \prime}(1)+z_{x x}(1) f^{\prime}(1)=0 \\
f^{\prime \prime \prime}(1)+\frac{3 z_{x x}(1)}{z_{x}^{2}(1)} f^{\prime \prime}(1)+\frac{z_{x x x}(1)}{z_{x}^{3}(1)} f^{\prime}(1)-\frac{(\alpha+\lambda \beta)}{z_{x}^{3}(1) E I(1)} f(1)=0
\end{array}\right.
$$

with

$$
\begin{aligned}
a(z) & =\frac{6 z_{x x}}{z_{x}^{2}}+\frac{2 E I^{\prime}(x)}{z_{x} E I(x)} \\
b(z) & =\frac{3 z_{x x}^{2}}{z_{x}^{4}}+\frac{6 z_{x x} E I^{\prime}(x)}{z_{x}^{3} E I(x)}+\frac{E I^{\prime \prime}(x)}{z_{x}^{2} E I(x)}+\frac{4 z_{x x x}}{z_{x}^{3}} \\
c(z) & =\frac{z_{x x x x}}{z_{x}^{4}}+\frac{2 z_{x x x} E I^{\prime}(x)}{z_{x}^{4} E I(x)}+\frac{z_{x x} E I^{\prime \prime}(x)}{z_{x}^{4} E I(x)} \\
z_{x} & =\frac{1}{p}\left(\frac{m(x)}{E I(x)}\right)^{\frac{1}{4}}, z_{x}^{4}=\frac{1}{p^{4}} \frac{m(x)}{E I(x)}
\end{aligned}
$$

and

$$
z_{x x}=\frac{1}{4 p}\left(\frac{m(x)}{E I(x)}\right)^{\frac{-3}{4}} \frac{d}{d x}\left(\frac{m(x)}{E I(x)}\right)^{\frac{1}{4}}
$$

The equation in (16) is

$$
\begin{gathered}
f^{(4)}(z)+a(z) f^{\prime \prime \prime}(z)+b(z) f^{\prime \prime}(z)+c(z) f^{\prime}(z)+\lambda^{2} h^{4} f(z)=0 \\
0<z<1 .
\end{gathered}
$$

This can be further implied by applying another invertible transformation:

$$
g(z)=\exp \left(\frac{1}{4} \int_{0}^{z} a(\zeta) d \zeta\right) f(z), \quad 0<z<1,
$$

and we arrive at the following eigenvalue problem that is equivalent to the 
original one for all $0<z<1$ :

$$
\left\{\begin{array}{l}
g^{(4)}(z)+a_{1}(z) g^{\prime \prime}(z)+a_{2}(z) g^{\prime}(z)+a_{3} g(z)+\lambda^{2} p^{4} g(z)=0, \\
g(0)=g^{\prime}(0)=0, \\
g^{\prime \prime}(1)+a_{11} g^{\prime}(1)+a_{12} g(1)=0, \\
g^{\prime \prime \prime}(1)+a_{21} g^{\prime \prime}(1)+a_{22} g^{\prime}(1)+a_{23} g(1)=0,
\end{array}\right.
$$

where

$$
\begin{aligned}
& a_{1}(z)=-\frac{3}{2} a^{\prime}(z)-\frac{3}{8} a^{2}(z)+b(z), \\
& a_{2}(z)= \frac{1}{8} a^{3}(z)-\frac{1}{2} a(z) b(z)-a^{\prime \prime}(z)+c(z), \\
& a_{3}(z)= \frac{3}{16} a^{\prime 2}(z)-\frac{1}{4} a^{\prime \prime \prime}(z)+\frac{3}{32} a^{\prime}(z) a^{2}(z)-\frac{3}{256} a^{4}(z) \\
&+b(z)\left(\frac{1}{16} a^{2}(z)-\frac{1}{4} a^{\prime}(z)\right)-\frac{a(z) c(z)}{4}, \\
& a_{11}=-\frac{1}{2} a(1)+\frac{z_{x x}(1)}{z_{x}^{2}(1)}, \\
& a_{12}= \frac{1}{16} z_{x}^{2}(1) a^{2}(1)-\frac{1}{4} z_{x}^{2}(1) a^{\prime}(1)-\frac{1}{4} z_{x x}(1) a(1) \\
& z_{x}^{2}(1) \\
& a_{21}=-\frac{3}{4} a(1)+\frac{3 z_{x x}(1)}{z_{x}^{2}(1)}, \\
& a_{22}=-\frac{3}{4} a^{\prime}(1)+\frac{3}{16} a^{2}(1)-\frac{3 z_{x x}(1) a(1)}{2 z_{x}^{2}(1)}+\frac{z_{x x x}(1)}{z_{x}^{3}(1)}, \\
& a_{23}=-\frac{1}{4} a^{\prime \prime}(1)+\frac{3}{16} a^{\prime}(1) a(1)-\frac{1}{64} a^{3}(1)-\frac{3 z_{x x}(1) a^{\prime}(1)}{4 z_{x}^{2}(1)} \\
&+\frac{3 z_{x x}(1) a^{2}(1)}{16 z_{x}^{2}(1)}-\frac{3 z_{x x x}(1) a(1)}{4 z_{x}^{3}(1)}-\frac{(\alpha+\lambda \beta)}{z_{x}^{3}(1) E I(1)}, \\
& \theta_{0}-\frac{\lambda \beta}{z_{x}^{3}(1) E I(1)}, \text { with } \\
& \theta_{0}=-\frac{1}{4} a^{\prime \prime}(1)+\frac{3}{16} a^{\prime}(1) a(1)-\frac{1}{64} a^{3}(1)-\frac{3 z_{x x}(1) a^{\prime}(1)}{4 z_{x}^{2}(1)} \\
& a_{23} \frac{3 z_{x x}(1) a^{2}(1)}{16 z_{x}^{2}(1)}-\frac{3 z_{x x x}(1) a(1)}{4 z_{x}^{3}(1)}-\frac{\alpha}{z_{x}^{3}(1) E I(1)} . \\
&=
\end{aligned}
$$


Theorem 2. Let $\mathbb{A}$ be defined by (10) and (11), then an asymptotic expression of the eigenvalues of the problem (23) is given by

$$
\begin{aligned}
\lambda_{n}= & \frac{\sqrt{2}}{2 p^{2}}\left(\mu_{3}-\mu_{2}\right) \pm \frac{1}{p^{2}}\left[\frac{\sqrt{2}}{2}\left(\mu_{3}+\mu_{2}\right)+\left(n+\frac{1}{2}\right)^{2} \pi^{2}\right] i \\
& +O\left(\frac{1}{n}\right)
\end{aligned}
$$

where $n=N, N+1, \ldots$ with $N$ large enough, and

$$
\begin{aligned}
\mu_{3}-\mu_{2} & =2 \sqrt{2} \frac{\beta}{z_{x}^{3}(1) E I(1) p^{2}}=-\frac{2 \sqrt{2} \beta p}{E I(1)}\left(\frac{m(1)}{E I(1)}\right)^{\frac{-3}{4}} \\
\mu_{3}+\mu_{2} & =2 \sqrt{2}\left(\mu_{1}+b_{11}\right) .
\end{aligned}
$$

Moreover, $\lambda_{n}(n=N, N+1, \ldots)$ with sufficiently large modulus are simple and distinct except for finitely many of them, and satisfy

$$
\lim _{n \rightarrow+\infty} \operatorname{Re}\left(\lambda_{n}\right)=-\frac{2 \beta}{p E I(1)}\left(\frac{m(1)}{E I(1)}\right)^{\frac{-3}{4}} .
$$

Proof. (See [13]).

\section{Finite Differences Method}

In this section, we use the finite differences method to study numerically the spectrum of operator of the problem (3), see P.G. Ciarlet [3], J. Rappaz and M. Picasso [11]. Then, we apply QZ method, see G.H. Golub [7], C.B. Moler and G.W. Stewart [9]. Finally, we study the influence of parameters $\alpha, \beta$ in velocity convergence of the system (3). The length of the beam is chosen to be unity, $E I(x)=(1+x)^{4}$, is the stiffness of the beam, and $m(x)=(1+x)^{2}$ is the mass density.

In the rest of this section we denote $u$ by $g$ and $x$ by $z(x)$ in system $(23)$. 
Hence we get the following system $0<x<1$ :

$$
\left\{\begin{array}{l}
u^{(4)}(x)+a_{1}(x) u^{\prime \prime}(x)+a_{2}(x) u^{\prime}(x)+a_{3}(x) u(x) \\
+\lambda^{2} p^{4} u(x)=0, \\
u(0)=u^{\prime}(0)=0, \\
u^{\prime \prime}(1)+a_{11} u^{\prime}(1)+a_{12} u(1)=0, \\
u^{\prime \prime \prime}(1)+a_{21} u^{\prime \prime}(1)+a_{22} u^{\prime}(1)+a_{23} u(1)=0 .
\end{array}\right.
$$

We develop a numerical scheme based on the finite differences method for the eigenvalue problem (27) associated with the evolutive system defined by (3). In practical, the spectral problem is not simple and cannot be solved by formula. Even when there is a formula, it might be so complicated that we would prefer to visualize the eigenvalues by looking at a graph. The finite differences method is one of the best known of the most important techniques of computation using quite simple equations and consists of replacing each derivative by a difference quotient. Consider for instance, a function $u: x \mapsto u(x)$ of variable $x$. Choose a mesh size $h=\frac{1}{n}$ for all $n \in \mathbb{N}^{*}$. We approximate the value $u\left(x_{i}\right)$ for $x_{i}=i h, i=0,1, \ldots, n$ and $x_{0}=0, x_{n}=1$, by a number $u_{i}$ indexed by an integer $i: u_{i} \sim u\left(x_{i}\right)$. Using Taylor expansions, we get for the derivatives, the following approximations for $i=2, \ldots, n-2$ :

$$
\begin{aligned}
u^{\prime}\left(x_{i}\right)= & \frac{-3 u\left(x_{i}\right)+4 u\left(x_{i}+h\right)-u\left(x_{i}+2 h\right)}{2 h}+O\left(h^{2}\right), \\
u^{\prime \prime}\left(x_{i}\right)= & \frac{u\left(x_{i}-h\right)-2 u\left(x_{i}\right)+u\left(x_{i}+h\right)}{h^{2}}+O\left(h^{2}\right), \\
u^{(4)}\left(x_{i}\right)= & \frac{u\left(x_{i}-2 h\right)-4 u\left(x_{i}-h\right)+6 u\left(x_{i}\right)}{h^{4}}+ \\
& \frac{-4 u\left(x_{i}+h\right)+u\left(x_{i}+2 h\right)}{h^{4}}+O\left(h^{2}\right) .
\end{aligned}
$$

The approximations of the boundary conditions give:

$$
u_{0}=0, \quad 4 u_{1}-u_{2}=0,
$$

where $u\left(x_{i}\right)=u_{i}$, for $i=0,1, \ldots, n$.

Expanding the function $u: x \mapsto u(x)$ according to its Taylor series of order 4, we get:

$$
u(1-h)=u(1)-h u^{\prime}(1)+\frac{h^{2}}{2 !} u^{\prime \prime}(1)-\frac{h^{3}}{3 !} u^{\prime \prime \prime}(1)+\frac{h^{4}}{4 !} u^{(4)}\left(\xi_{1}\right),
$$




$$
\begin{gathered}
u(1-2 h)=u(1)-2 h u^{\prime}(1)+2 h^{2} u^{\prime \prime}(1)-\frac{8 h^{3}}{3 !} u^{\prime \prime \prime}(1)+\frac{16 h^{4}}{4 !} u^{(4)}\left(\xi_{2}\right), \\
u(1-3 h)=u(1)-3 h u^{\prime}(1)+\frac{9 h^{2}}{2 !} u^{\prime \prime}(1)-\frac{27 h^{3}}{3 !} u^{\prime \prime \prime}(1)+\frac{81 h^{4}}{4 !} u^{(4)}\left(\xi_{3}\right),
\end{gathered}
$$

with $\left.\xi_{j} \in\right] 1-j h, 1[, j=1,2,3$.

We eliminate $u^{\prime \prime}(1)$ (respectively $\left.u^{\prime}(1)\right)$ in equations (32) and (33) we get:

$$
\begin{aligned}
u^{\prime}(1) & =\frac{u(1-2 h)-4 u(1-h)+3 u(1)}{2 h}+O\left(h^{2}\right), \\
u^{\prime \prime}(1) & =\frac{u(1-2 h)-2 u(1-h)+u(1)}{h^{2}}+O\left(h^{2}\right) .
\end{aligned}
$$

We eliminate $u^{\prime}(1)$ in equations (32), (33) and (34) we get:

$$
u^{\prime \prime \prime}(1) \simeq \frac{-u(1-3 h)+3 u(1-2 h)-3 u(1-h)+u(1)}{3 h^{3}} .
$$

The approximation of the system (27) is:

$$
\left\{\begin{array}{l}
u_{i-2}+a_{i} u_{i-1}+\left(b_{i}+\lambda^{2} p^{4} h^{4}\right) u_{i}+c_{i} u_{i+1}+d_{i} u_{i+2}=0 \\
i=2, \ldots, n-2 \\
u_{0}=4 u_{1}-u_{2}=0 \\
\left(2+h a_{11}\right) u_{n-2}-\left(4+4 h a_{11}\right) u_{n-1}+\left(2+3 h a_{11}+2 a_{12} h^{2}\right) u_{n}=0 \\
-2 u_{n-3}+\left(6+6 a_{21} h+3 a_{22} h^{2}\right) u_{n-2}+\left(-6-12 a_{21} h-12 a_{22} h^{2}\right) u_{n-1} \\
+\left(2+6 a_{21} h+9 a_{22} h^{2}+3 \theta_{0} h^{3}-\frac{3 \lambda \beta h^{3}}{z_{x}^{3}(1) E I(1)}\right) u_{n}=0
\end{array}\right.
$$

where

$$
\begin{aligned}
a_{i} & =-4+a_{1 i} h^{2}, \\
b_{i} & =6-2 h^{2} a_{1 i}-\frac{3}{2} a_{2 i} h^{3}+a_{3 i} h^{4}, \\
c_{i} & =-4+a_{1 i} h^{2}+2 a_{2 i} h^{3}, d_{i}=1-\frac{a_{1 i}}{2} h^{3}, \\
\theta_{0} & =a_{23}+\frac{\lambda \beta h^{3}}{z_{x}^{3}(1) E I(1)},
\end{aligned}
$$

with 


$$
a_{1 i}=a_{1}\left(x_{i}\right), \quad a_{2 i}=a_{2}\left(x_{i}\right) \text { and } a_{3 i}=a_{3}\left(x_{i}\right), \quad i=2, \ldots, n-2 .
$$

The system (38) can be written following form:

$$
\left\{\begin{array}{l}
\lambda^{2} u_{1}-\frac{1}{4} \lambda^{2} u_{2}=0, \\
u_{i-2}+a_{i} u_{i-1}+\left(b_{i}+\lambda^{2} p^{4} h^{4}\right) u_{i}+c_{i} u_{i+1}+d_{i} u_{i+2}=0, \\
i=2, \ldots, n-2, \\
\left(2+h a_{11}\right) u_{n-2}-\left(4+4 h a_{11}\right) u_{n-1}+\left(2+3 h a_{11}+2 a_{12} h^{2}\right) u_{n}=0, \\
-2 u_{n-3}+\left(6+6 a_{21} h+3 a_{22} h^{2}\right) u_{n-2}+\left(-6-12 a_{21} h-12 a_{22} h^{2}\right) u_{n-1} \\
+\left(2+6 a_{21} h+9 a_{22} h^{2}+3 \theta_{0} h^{3}-\frac{3 \lambda \beta h^{3}}{z_{x}^{3}(1) E I(1)}\right) u_{n}=0 .
\end{array}\right.
$$

We can calculate $u_{1}, \ldots, u_{n}$ using the scheme for the partial differential equation. Here the finite differences method looks for the complex number $\lambda$ such as there exists a nonzero vector

$U=\left(u_{1}, u_{2}, \ldots, u_{n}\right)^{T}$ satisfies the above discrete problem (43). Now, we consider the matrices $A, B, C$ of order $n$, defined as follows:

$$
\begin{gathered}
A_{i j}=\left\{\begin{array}{ccc}
1 & \text { for } & i=j=1, \\
-\frac{1}{4} & \text { for } & i=1, j=2, \\
p^{4} h^{4} & \text { for } & i=j=2, \ldots, n-2, \\
0 & \text { elsewhere, }
\end{array}\right. \\
B_{i j}=\left\{\begin{array}{ccc}
-\beta h^{3} \frac{3}{z_{x}^{3}(1) E I(1)} & \text { for } & i=j=n, \\
0 & \text { for } & i=j=1, \ldots, n-1, \\
0 & \text { elsewhere, }
\end{array}\right.
\end{gathered}
$$




$$
\begin{aligned}
& C_{i j}=\left\{\begin{array}{lll}
b_{i} & \text { if } \quad i=j, \\
a_{i} & \text { if } \quad j=i-1, \\
c_{i} & \text { if } \quad j=i+1, \\
d_{i} & \text { if } \quad j=i+2, \\
1 & \text { if } \quad j=i-2 .
\end{array}\right. \\
& \left\{\begin{aligned}
C_{11} & =0, \\
C_{12} & =0, \\
C_{n-1 n-2} & =2+h a_{11}, \\
C_{n-1 n-1} & =-4-4 h a_{11} \\
C_{n-1 n} & =2+3 h a_{11}+2 a_{12} h^{2}, \\
C_{n n-3} & =-2, \\
C_{n n-2} & =6+2 h a_{21}+a_{22} h^{2}, \\
C_{n n-1} & =-6-12 h a_{21}-12 a_{22} h^{2}, \\
C_{n n} & =2+6 h a_{21}+9 a_{22} h^{2}+3 \theta_{0} .
\end{aligned}\right.
\end{aligned}
$$

The problem (43) takes the following equation form:

$$
\lambda^{2} A U+\lambda B U+C U=0
$$

where the matrices $A, B$, and $C$ are defined as above, and where

$$
U=\left(u_{1}, u_{2}, \ldots, u_{n}\right)^{T}
$$

Now, we introduce the auxiliary vector:

$$
Z=\lambda U
$$

and we get the system: 


$$
\left\{\begin{aligned}
A Z & =\lambda A U \\
-C U & =\lambda A Z+\lambda B U,
\end{aligned}\right.
$$

which is equivalent to the system:

$$
\left(\begin{array}{cc}
A & 0 \\
0 & -C
\end{array}\right)\left(\begin{array}{l}
Z \\
U
\end{array}\right)=\lambda\left(\begin{array}{ll}
0 & A \\
A & B
\end{array}\right)\left(\begin{array}{l}
Z \\
U
\end{array}\right)
$$

We get a generalized eigenvalue problem:

$$
M V=\lambda N V
$$

with

$$
M=\left(\begin{array}{cc}
A & 0 \\
0 & -C
\end{array}\right), \quad N=\left(\begin{array}{cc}
0 & A \\
A & B
\end{array}\right) \text { and } V=(Z, U)^{T} .
$$

And we use QZ method to resolve this problem.

\section{Numerical Experiments}

To evaluate the effect of parameters $\alpha$ and $\beta$ on the spectrum, we give here in the same field, the graphs of the spectrum for different values of the control in position $\alpha$ and velocity $\beta$.

We do the study for three cases:

First case : $\alpha=0$

a) We take $\beta \in] 0 ; 1[$.

We observe that when the parameter $\beta \in] 0 ; 1[$ without control in position $\alpha$, the location of spectrum moves rapidly on the left-hand side of the complex plane.

b) We take $\beta \geq 1$.

We observe that when the parameter $\beta$ increases without control in position $\alpha$, the location of spectrum moves rapidly on the left-hand side of the complex plane.

Second case $\alpha>0$ :

a) $\beta \in] 0 ; 1[$. We fix $\alpha=10$ and take $\beta=0.1$ and $\beta=0.3$.

We observe that when the parameter $\beta \in] 0 ; 1[$ for a fixed value of $\alpha>0$, the location of spectrum moves rapidly on the left-hand side of the complex plane.

b) $\beta \geq 1$. We fix $\alpha=10$ and take $\beta=1$ and $\beta=10$. 


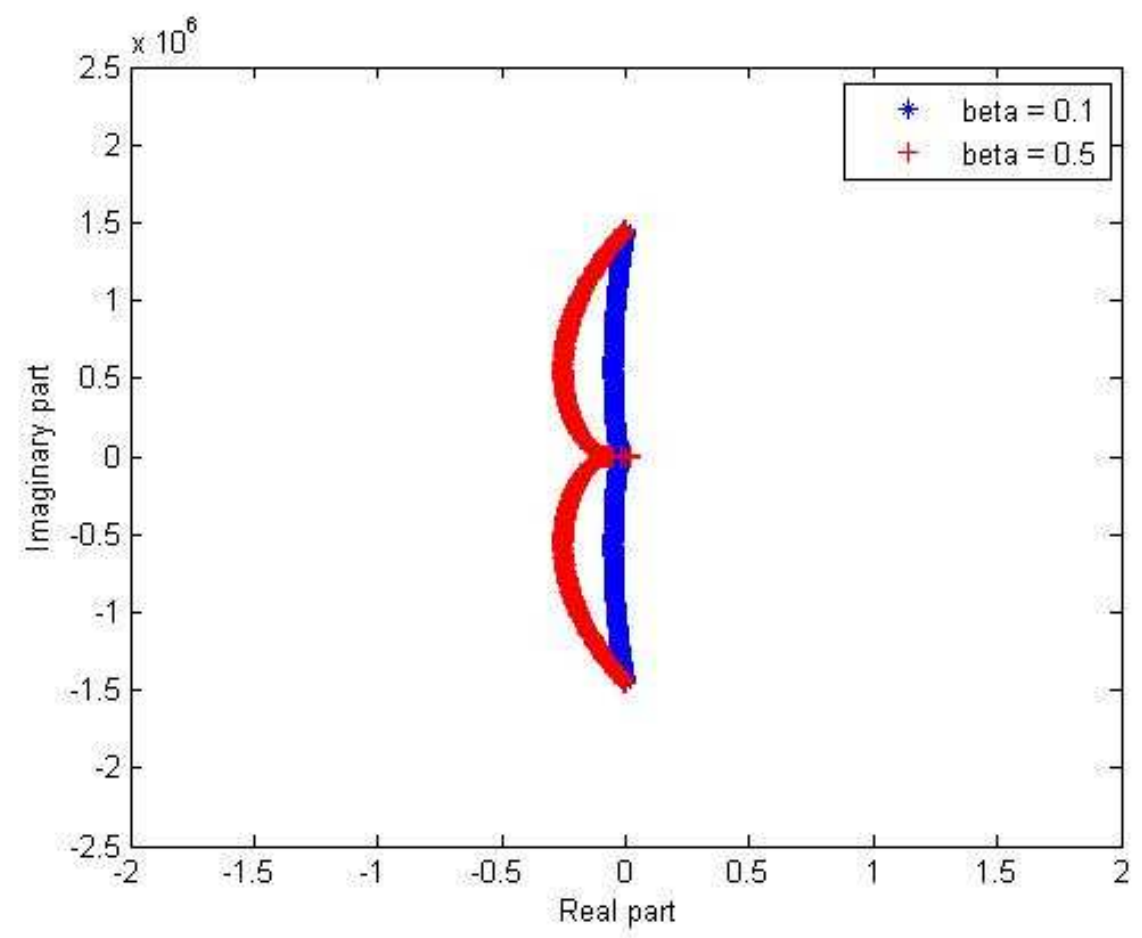

Figure 1: Effect of parameter $\beta \in] 0 ; 1[$ on the spectrum for $\alpha=0$.

We observe that when the parameter $\beta$ increases for a fixed value of $\alpha>0$, the location of spectrum moves rapidly on the left-hand side of the complex plane.

\section{Third case:}

We fixe $\beta=10$ and take $\alpha=10$ and $\alpha=20$.

We observe that the parameter $\alpha$ has not effect on the spectrum for a fixed value of $\beta$ and the location of spectrum stays on the left-hand side of the complex plane.

Conclusions. From all previous observations we claim that the control feedback $\alpha$ in position has no influence on the optimal decay rate of the energy. But the control feedback in velocity improves the optimal decay of the energy. At last, in [2] the author has theoretically proved that the control in velocity was enough to establish the system exponential stability when $\alpha=0$, which corresponds to our numerical results. Finally the numerical method thus set, 


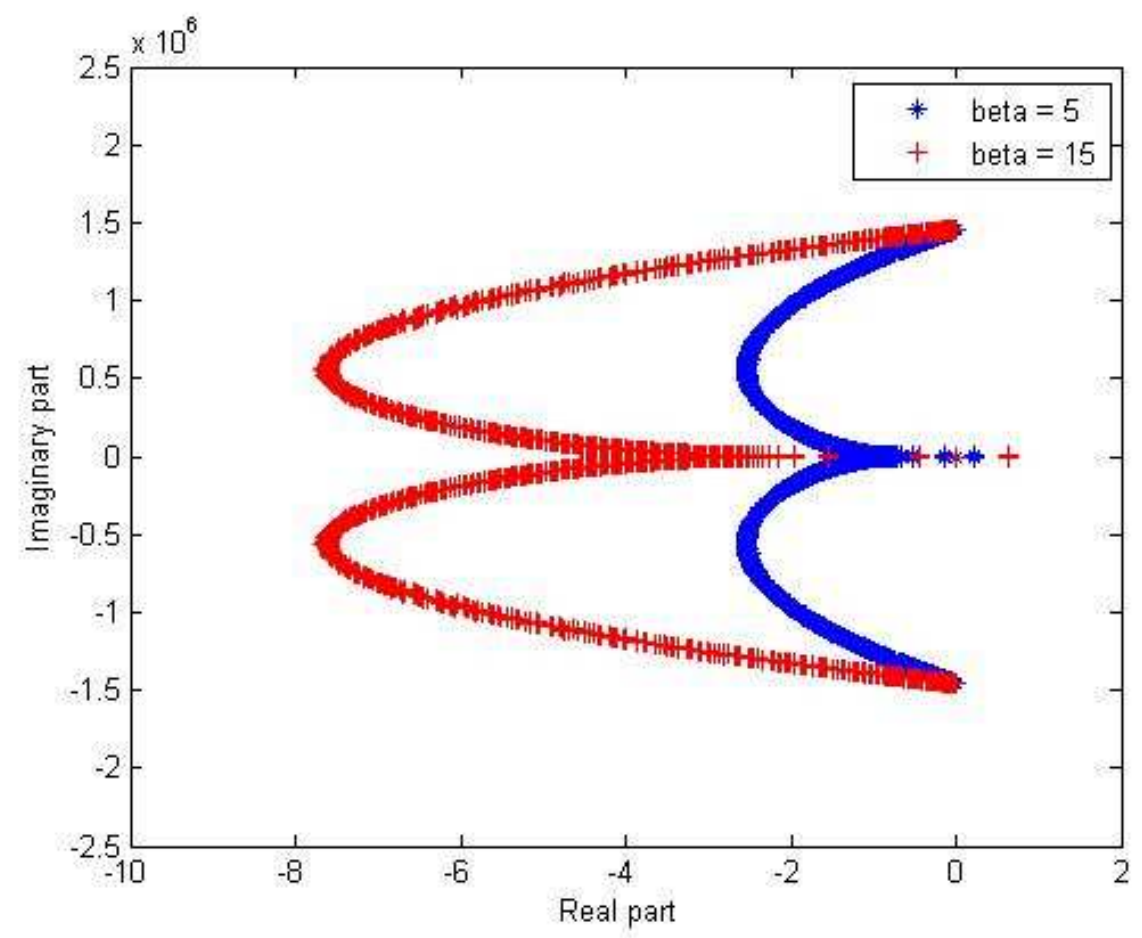

Figure 2: Effect of parameter $\beta \geq 1$ on the spectrum for $\alpha=0$.

permits to appreciate the impact of the control force in position on the optimal decay rate of the studied system energy.

\section{References}

[1] M.D. Aouragh and N. Yebari, Stabilisation exponentielle d'une équation des poutres d'Euler-Bernoulli à coefficients variables, Annales Mathématiques Blaise Pascal, 16, No. 2 (2009), 483-510.

[2] B.Z. Guo, Riesz basis property and exponential stability of controlled Euler-Bernoulli beam equation whith variable coefficients, SIAM J. Control Optim., 40, No. 6 (2002), 1905-1923.

[3] P.G. Ciarlet, Introduction à l'analyse numérique matricielle et à l'optimisation, Ed. Masson (1982). 


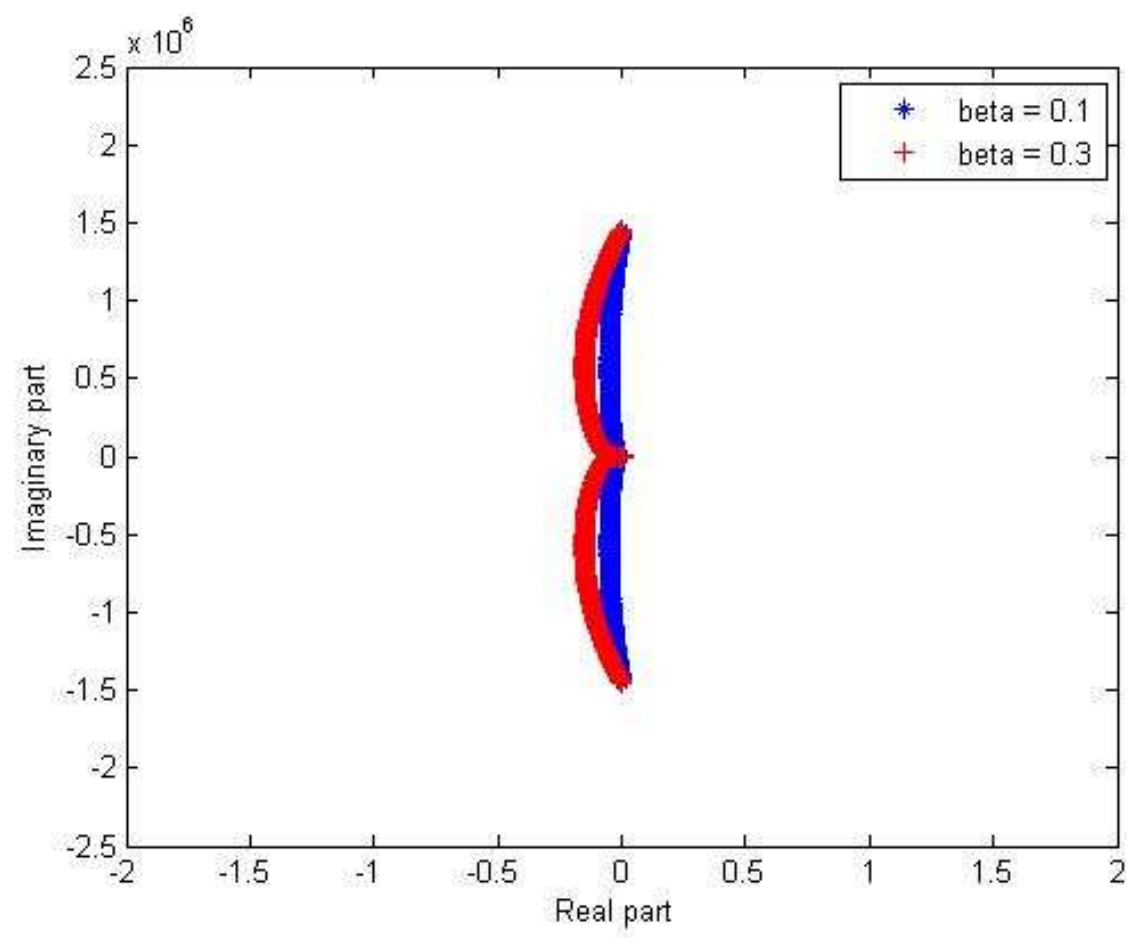

Figure 3: Effect of parameter $\beta \in] 0 ; 1[$ on the spectrum for $\alpha=10$.

[4] M. Cherkaoui, F. Conrad, N. Yebari, Optimal decay rate of energy for wave equation with boundary feedback, Advances in Mathematical Sciences and Applications, 12 (2002), 549-568.

[5] S. Cox, E. Zuazua, The rate at which energy decays in a damping string, Comm. Partial Differential Equations, 19 (1994), 213-243.

[6] R.F. Curtain and H.J. Zwart, An Introduction to Infinite Dimensional Linear System Theory, Springer Verlag, New York (1995).

[7] G.H. Golub and C.F.V. Loan, Matrix Computations, The Johns Hopkins University Press (1989).

[8] E.P. Mensah, K.A. Touré and M.M. Taha, Numerical approximation of the spectrum for a hyperbolic equation with boundary condition, Far East Journal of Applied Mathematics, 60, No. 1 (2011), 41-53. 


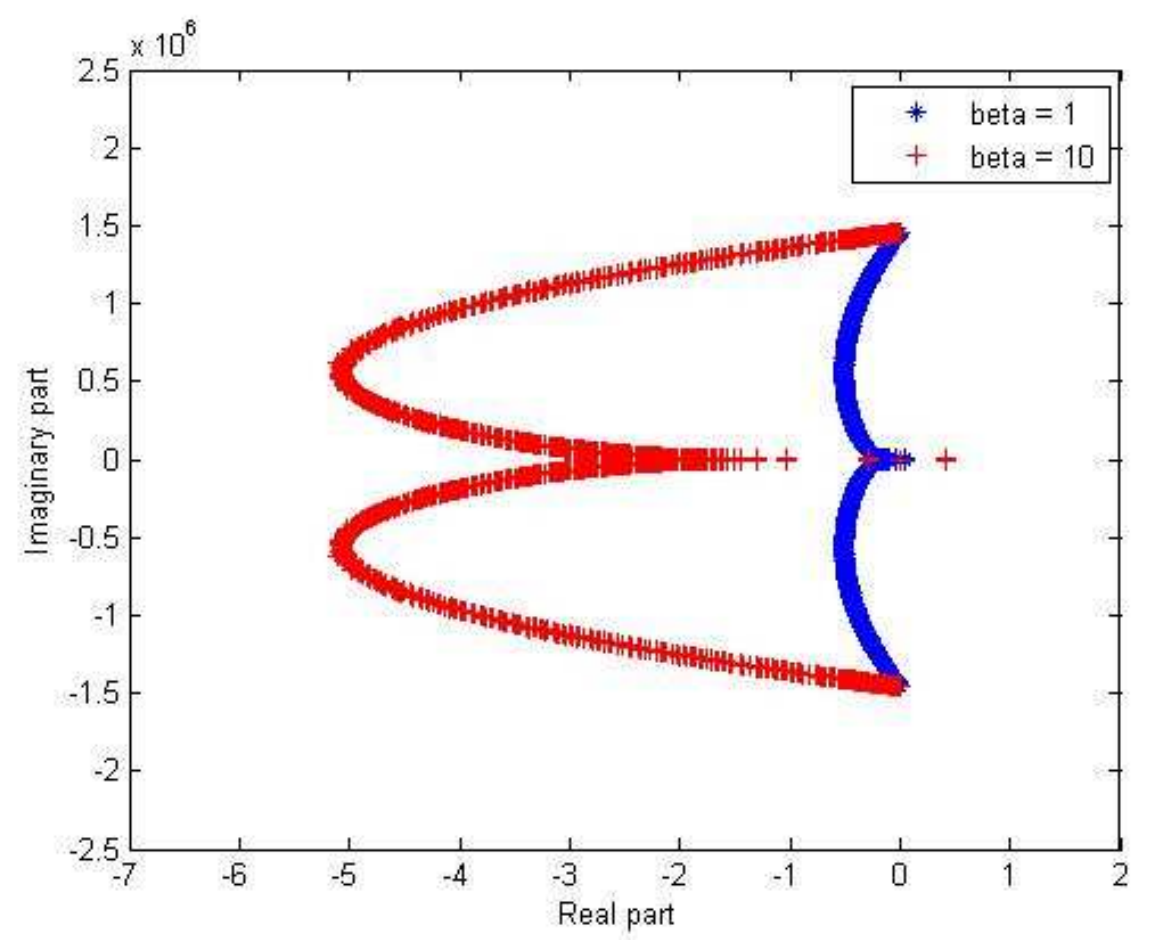

Figure 4: Effect of parameter $\beta \geq 1$ on the spectrum for $\alpha=10$.

[9] C.B. Moler and G.W. Stewart, An algorithm for generalized matrix eigenvalue problems, SIAM J. Numer. Anal. 10 (1973), 241-256 (Collection of articles dedicated to the memory of George E. Forsythe).

[10] Ö. Morgül, A dynamic control law for the wave equation, Automatic, 30, No. 11 (1994), 1785-1792.

[11] J. Rappaz and M. Picasso, Introduction à l'analyse numérique, Press Polytechniques et Universitaires, Lausanne (1998).

[12] P. Rideau, Contrôle d'assemblage de poutres flexibles par des capteurs actionneurs ponctuels: étude du spectre du système, Thèse, École Nationale Supérieure des Mines de Paris, Sophia-Antipolis (1985).

[13] K.A. Touré, A. Coulibaly and A.A.H. Kouassi, Riesz basis, exponential stability of variable coefficients Euler-Bernoulli beams with a force control 


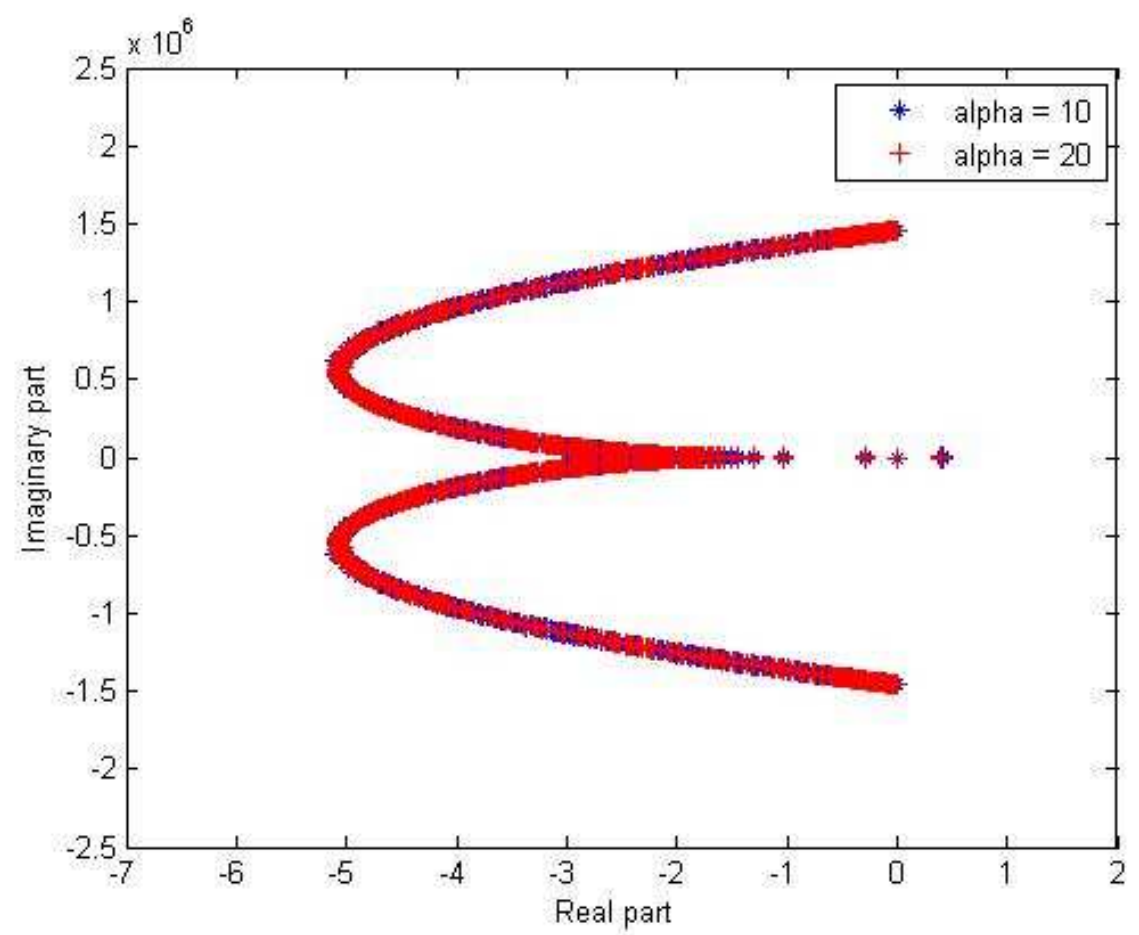

Figure 5: Effect of parameter $\alpha$ on the spectrum for $\beta$ fixed.

in position and vellocity. Far East Journal of Applied Mathematics, 88, No. 3 (2014), 193-220.

[14] K.A. Touré, E. Mensah and M. Taha, On the stabilization of a flexible cable with boundary feedback, Communications in Mathematics and Applications, 2, Nos 2-3 (2011), 111-129. 